\title{
“Balloon pulmonary angioplasty for the treatment of chronic thromboembolic pulmonary hypertension: is Europe behind?" Irene M. Lang and Hiromi Matsubara. Eur Respir J 2019; 53: 1900843.
}

\section{CrossMark}

The above-mentioned editorial has been corrected and republished online; the amendments have been discussed in the correspondence articles published in this issue of the European Respiratory Journal $[1,2]$. The sentence that originally read:

"While there are currently 1.7 surgical pulmonary endarterectomies per million of population performed in Europe annually, compared with 0.9 per million in the USA, only approximately 60 pulmonary endarterectomies are performed annually in Japan, accounting for one procedure per billion population."

has been corrected to:

"While there are currently 1.7 surgical pulmonary endarterectomies per million of population performed in Europe annually, compared with 0.9 per million in the USA, only approximately 60 pulmonary endarterectomies are performed annually in Japan, accounting for 0.47 procedures per million population."

The table has also been amended and re-formatted for clarity. The new version is reproduced below.

\begin{tabular}{|c|c|c|c|c|c|c|}
\hline Total patients $\mathbf{n}$ & \multicolumn{2}{|c|}{184} & \multicolumn{2}{|c|}{56} & \multicolumn{2}{|c|}{308} \\
\hline Age years & \multicolumn{2}{|c|}{$63 \pm 14$} & \multicolumn{2}{|c|}{$65(55-74)$} & \multicolumn{2}{|c|}{$62 \pm 13$} \\
\hline Females $\%$ & \multicolumn{2}{|c|}{49} & \multicolumn{2}{|c|}{61} & \multicolumn{2}{|c|}{80} \\
\hline Total procedures performed $n$ & \multicolumn{2}{|c|}{$\begin{array}{c}1006 \\
5 \text { (median) }\end{array}$} & \multicolumn{2}{|c|}{$\begin{array}{c}266 \\
5 \text { (median) }\end{array}$} & \multicolumn{2}{|c|}{$\begin{array}{c}1408 \\
4 \text { (median) }\end{array}$} \\
\hline & Baseline & After BPA & Baseline & After BPA & Baseline & After BPA \\
\hline Patients n & \multicolumn{2}{|c|}{154} & 56 & 55 & 308 & 196 \\
\hline 6MWD m & $396 \pm 120$ & $441 \pm 104$ & $358 \pm 108$ & $391 \pm 108$ & $318 \pm 122$ & $430 \pm 109$ \\
\hline mPAP $\mathrm{mmHg}$ & $43.9 \pm 9.5$ & $31.6 \pm 9.0$ & $40 \pm 12$ & $33 \pm 11$ & $43.2 \pm 11.0$ & $22.5 \pm 5.4$ \\
\hline$P_{\mathrm{aO}_{2}} \mathrm{mmHg}$ & $65.0 \pm 9.0$ & $73.3 \pm 12.0$ & $62.0 \pm 9.0$ & $66.0 \pm 10.0$ & Not stated & Not stated \\
\hline Patients on riociguat and/or drugs approved for PAH \% & 62 & Not stated & 92 & Not stated & 72 & 45 \\
\hline Lung injury ( $\%$ of sessions) & \multicolumn{2}{|c|}{9.1} & \multicolumn{2}{|c|}{9.4} & \multicolumn{2}{|c|}{17.8} \\
\hline 30-day mortality \% & \multicolumn{2}{|c|}{2.2} & \multicolumn{2}{|c|}{1.8} & \multicolumn{2}{|c|}{2.6} \\
\hline
\end{tabular}

Data are presented as mean \pm SD or median (interquartile range), unless stated otherwise. 6MWD: 6-min walk distance; mPAP: mean pulmonary artery pressure; $P_{\mathrm{aO}_{2}}$ : arterial oxygen tension; $\mathrm{S}_{\mathrm{aO}_{2}}$ : arterial oxygen saturation; $\mathrm{CO}$ : cardiac output; $\mathrm{Cl}$ : cardiac index; PVR: pulmonary vascular resistance; $\mathrm{PAH}$ : pulmonary arterial hypertension.

\section{References}

1 Jais $\mathrm{X}$, Brenot $\mathrm{P}$, Montani $\mathrm{D}$, et al. A roadmap for management of chronic thromboembolic pulmonary hypertension. Eur Respir J 2019; 54: 1901295

2 Lang IM, Matsubara H. Balloon pulmonary angioplasty for the treatment of chronic thromboembolic pulmonary hypertension: is Europe behind? Eur Respir J 2019; 54: 1901639. 